\title{
DISTRIBUTED INTERCULTURAL PROJECT-BASED LEARNING - A NOVEL APPROACH - EXPERIENCES FROM A QUADRILATERAL INTERDISCIPLINARY COLLABORATION
}

\author{
Manfred Meyer ${ }^{1}$, Agung Nugroho ${ }^{2}$, José Ochoa-Luna ${ }^{3}$, Colin Stanley ${ }^{4}$, \\ \& Heike Winschiers-Theophilus ${ }^{4}$ \\ ${ }^{1}$ Department of Mechanical Engineering, Westfälische Hochschule, Bocholt (Germany) \\ ${ }^{2}$ Department of Business Administration, Atma Jaya Catholic University of Indonesia (Indonesia) \\ ${ }^{3}$ Department of Computer Science, Universidad Católica San Pablo, Arequipa (Peru) \\ ${ }^{4}$ Faculty of Computing and Informatics, Namibia University of Science and Technology, Windhoek \\ (Namibia)
}

\begin{abstract}
This paper describes a new concept and experiences of a distributed interdisciplinary learning programme for students across continents. The aim is to provide students with a truly Global Intercultural Project Experience (GIPE) by working together with peers from around the world, and solving real-life client's problems. We have received seed-funding for four annual projects to engage students from Germany (Europe), Namibia (Africa), Indonesia (Asia), and Peru (Latin-America). In 2020, 30 students from four continents engaged in a one-semester distributed software development project for a Namibian client. Despite Covid-19 they successfully completed the project expressing deep appreciation for the learning opportunities overcoming challenges of working across wide-spread time zones, cultures, changing requirements, and various technical challenges. Considering the vast learning benefits, we suggest to incorporate such projects in all tertiary education curricula across the globe.
\end{abstract}

Keywords: Project-based learning, COIL, distributed software development, intercultural collaboration.

\section{Introduction}

Globalization requires Higher Education Institutions (HEIs) to provide relevant 21 st century skills and knowledge, while embracing digital transformation. The ongoing pandemic has fast-tracked a long-awaited educational transformation promoting distributed interdisciplinary on-line learning.

While Project-Based Learning (PBL) has been established since long and not only in HEIs as a student-centered approach where students acquire a deeper knowledge through active exploration of real-world challenges and problems (Bender, 2012), Collaborative Online International Learning (COIL) is nowadays receiving a growing interest as an innovative, cost-effective instructional method that promotes intercultural learning through online collaboration between faculty and students residing in different countries or locations within the context of a course (DePaul University, 2017). Although the idea of distributed student projects with online collaboration among different HEIs dates back to the late 1990s where it has already been successfully applied at least in the field of Software Engineering (Brereton, Gumbley \& Lees, 1998), the international and intercultural aspect came into focus only during the last decade (Appiah-Kubi \& Annan, 2020).

Although the concept of COIL is often being referred to as 'virtual mobility' only (SUNY COIL Center, n.d.), De Wit (2013) regards COIL "an important addition to the many forms of physical mobility" and Helm \& O'Dowd (2020) claim that it "should not be seen as being in competition with physical mobility, rather ... as a complement to physical mobility in blended mobility initiatives and as an inclusive, international learning opportunity for students and staff who may not be able to or want to have a physical or blended mobility." It is exactly this understanding that has led to our approach of enhancing the COIL setup by phases for short-term physical mobility to focus on a much denser intercultural exposure of students and staff in preparation for a successful online collaboration across continents and cultures.

\section{Background}

A quadrilateral partnership was conceptualized, building on long-term individual staff and institutional bilateral collaborations of the Westfälische Hochschule Bocholt with the Namibia University 
of Science and Technology, Windhoek, Atma Jaya Catholic University of Indonesia, Jakarta, and Universidad Católica San Pablo, Arequipa. The proposed programme was modelled on prior successfully completed bilateral student software development projects between German and Namibian students. Concerned with providing equal learning and traveling opportunities for all students and operating within funding options the programme incorporates traveling for all students; turn-taking client selections from the partner countries with an emphasis on interdisciplinary projects. The programme offers selected students the opportunity to gain a truly Global Intercultural Project Experience (GIPE) by working in a multicultural team on an international project. The main objective of the GIPE programme is to provide students with state-of-the art technical knowledge while working in a distributed multicultural team across continents. GIPE also aims at strengthening the collaboration among its partner universities and promoting intercultural exposure in general through 'internationalization@home' activities.

\section{The GIPE framework}

In an effort to strengthen internationalization efforts at German Universities of Applied Sciences, the German Academic Exchange Service (DAAD) has awarded a four year (2019-2023) funding for the GIPE framework programme. At the beginning representatives of all four Universities congregated in Germany to plan the implementation of the framework. A German representative then visited all partner Universities promoting GIPE at management level to ensure institutional commitment and support.

At the core of the GIPE framework are the annual student projects taking place from February to June/July (subject to different academic calendars and lecturing periods) proceeded by a client and project selection, evaluation of students' applications and awarding scholarships as well as requirements gathering and detailed project planning together with the selected client (see Figure 1).

Figure 1. The Masterplan for the GIPE Framework 2019-2023.

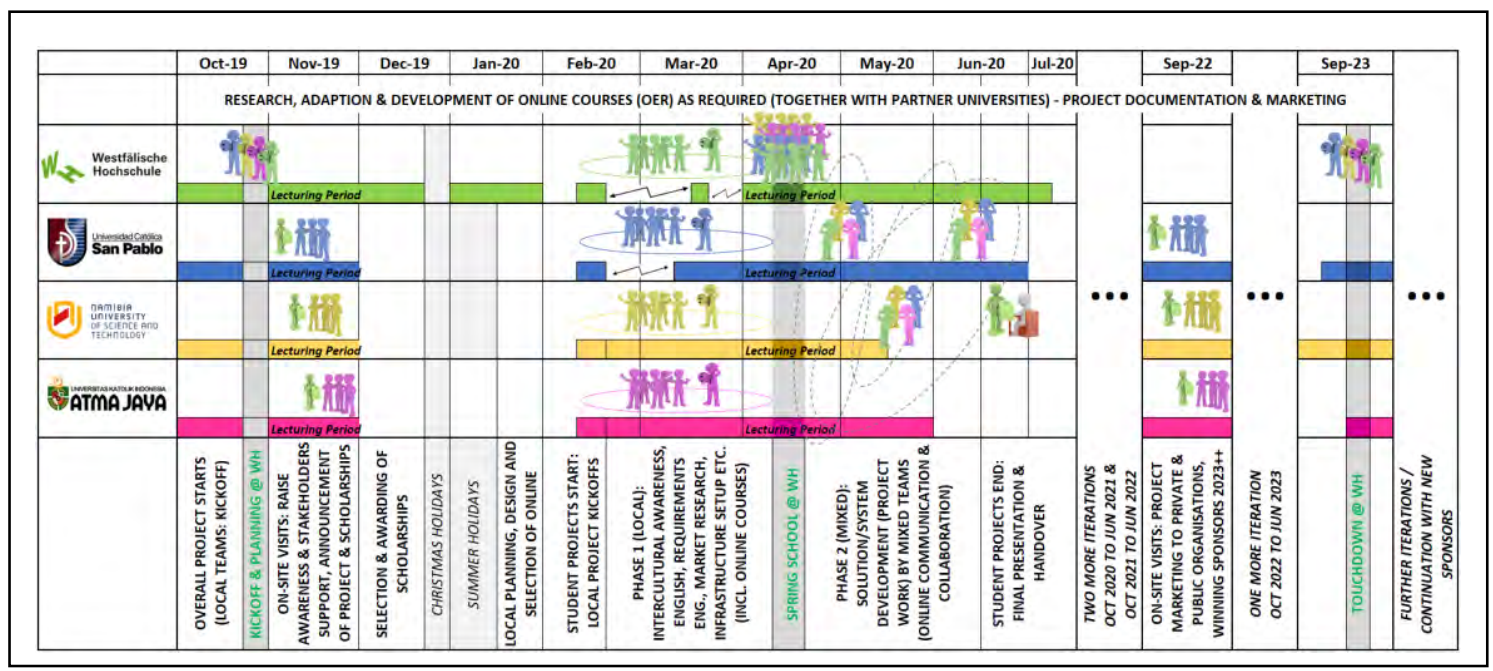

The annual GIPE projects consist of four phases:

1. On-line collaboration preparation: A virtual global kick-off event brings all stakeholders together. Students then get prepared for the various project tasks through targeted trainings. Students join the project in intervals depending on the home universities lecturing schedules.

2. Two-week face-to-face phase: All participating students and one representative lecturer from each university meet in Germany for team-building, intercultural exposure and mixed-team-setup ('Spring School').

3. On-line collaboration: the students continue working on their project tasks in mixed teams using various on-line collaboration tools.

4. One-week project-touchdown and hand-over: The German students travel to the client situated in Namibia, Peru or Indonesia (alternating) offering true international exposure.

To be noted is that the 2020 project was held entirely on-line due to the Covid-19 pandemic and the consequent travel restrictions.

\section{The GIPE 2020 project}

A diverse set of students was selected from each partner University prior to the start of the project. In Peru, four male and one female student joined, with one Business Administration and four Computer Science students (one master and three undergraduate students). In Indonesia, four male and one female student were chosen from the engineering discipline, i.e. electrical, mechanical and industrial 
engineering. In Germany, one female and four male students from IT-related and International Management programs (all Bachelor) and Mechanical Engineering (Master) were selected. In Namibia, the entire postgraduate Advanced Software Development class consisting of 13 students ( 2 female, 11 male) joined the project. Five travel grants were allocated based on the student's portfolio, however due to Covid-19 no student traveled. Each University had a dedicated lecturer overseeing their students, called guide. One part-time project coordinator situated in Germany was responsible for project communication and providing technology support.

A Namibian parastatal was identified as a suitable client, with whom high-level requirements were agreed upon prior to students joining the project. The task was to develop a national web portal to manage research projects, publications and researcher profiles. After signing off the requirement specification document and deciding on the most appropriate state-of-the-art technologies (mostly Software) to be used, the project was internally divided in six sub-projects, each assigned to one team of students: (1) Object Model, (2) Documentation, (3) Quality Control and Assurance, (4) Frontend, and (5) Business Logic. The allocation of students to teams was based on their country (mixed teams), their prior competencies as well as their interest. Thus, students filled in a skill competency survey, which was created by the project coordinator on the basis of needed competencies for the successful completion of this project. The students were then assigned ensuring skills matched, e.g. students who rated themselves very competent on user interface development such as HTML and JavaScript were assigned to the Frontend team. The skills competency survey also allowed the project coordinator to identify skills that were overall rated low yet needed. Thus, interventions were planned. For example, some students needed training on a concurrent version system (CVS) such as GitLab, which allows them to submit their work to a central repository and handle conflicts of files overwriting. Those students who were experienced using CVS systems conducted online tutorials showcasing the other students how to use GitLab.

The project progress was monitored on a weekly basis with meetings and presentations providing feedback. A variety of tools for collaboration and communication were used. For the core facilitation of the GIPE course content, Moodle was used. Slack was used for instant messaging, Slite was used for the overall project administration and Zoom for web meetings. The Object Team duties were adjusted towards the end of the project to focus on preparing deployment (handing over the developed application to the client) since their main task was a prerequisite for most of the other teams and was completed. The developed application (see interface in Figure 2), including the documentation, was handed over to the client, with a minimal delay. The client requested assistance with deployment as well as an extension of some functionalities. Thus, two Namibian students continued working on the project on the client-side, on a part-time basis.

Figure 2. The new STI web portal developed for NCRST.

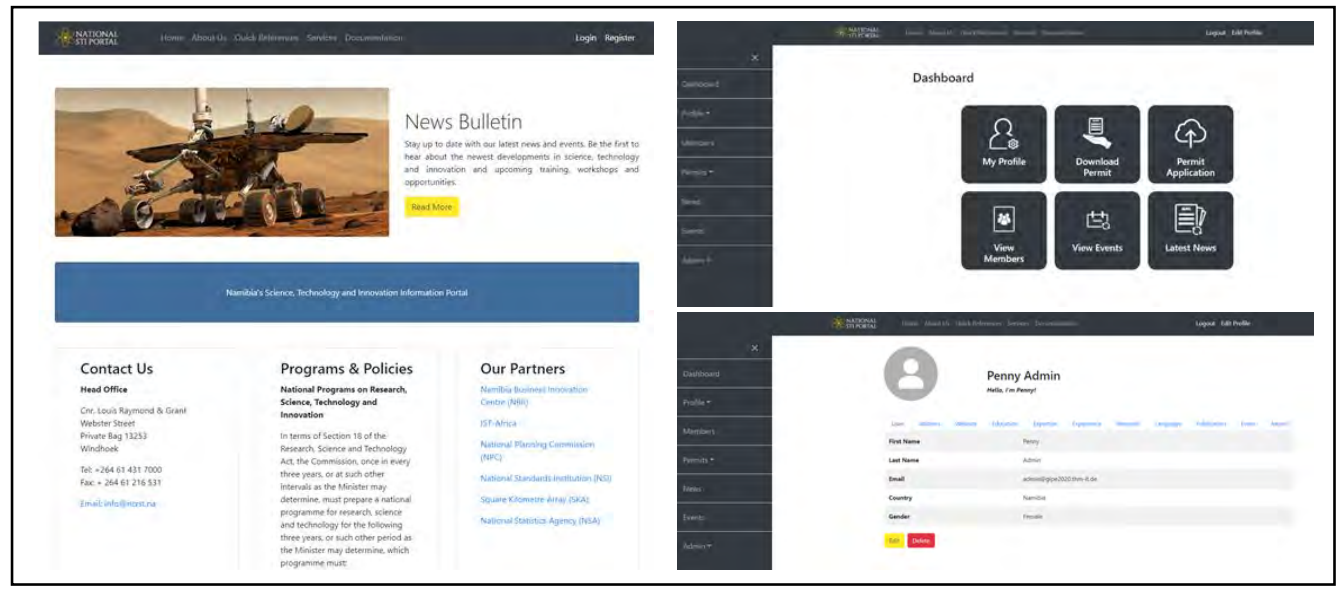

Each guide provided a performance feedback on each student's contribution to the success of the project. Each partner University further applied their own grading system to their students separately with credit points for passing/failing the course they registered for from their specific University. For example, in Namibia, each student was assessed on an individual project process report, a sub-project team deliverable (depending on the team a model, document, code, interface), a team presentation, and individual contribution.

\section{Student feedback}

Student feedback was provided on a voluntary basis by the students in form of videos or text at the end of the project, as well as formal challenges and reflections in the individual student process 
reports form by the Namibian students. It was observed that the Indonesian students provided much more details in their own language than in the English testimonials they also provided. At this point no systematic feedback analysis was undertaken but rather a search of personal statements confirming identified topics in the word frequency analysis. Figure 3 shows a word frequency word cloud, based on the video transcripts and written text feedback by the students, illustrating the most prominent topics such as project, experience, time, team, work, challenge.

Figure 3. Word Cloud generated from all testimonials from GIPE 2020 students.

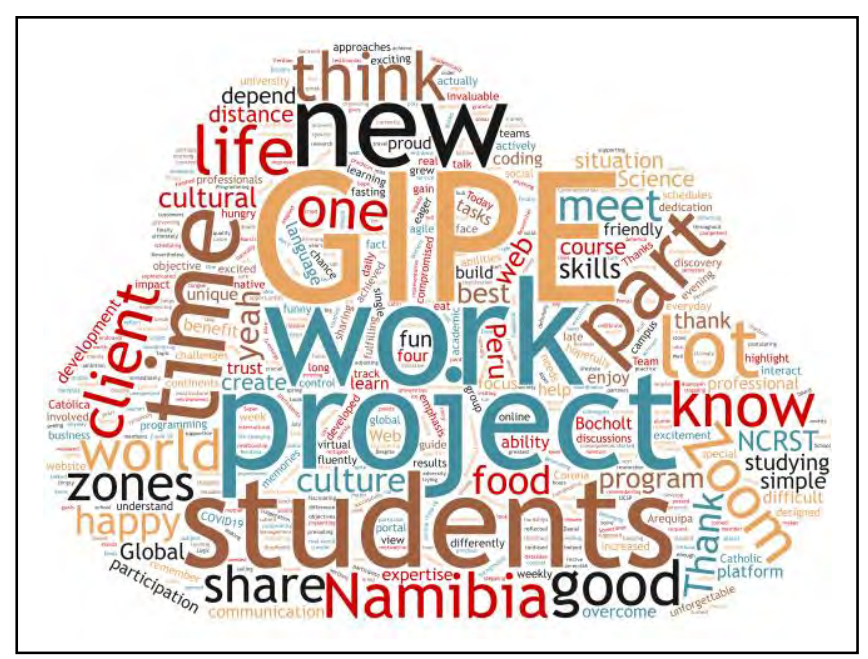

Related to the project work itself, the students appreciated the professional support provided by the project coordinator and the guides. An Indonesian student voiced that: " I appreciate this project and when I go through the trouble, all the guides will help me through many problems." A Namibian student added that "there was always support, it was as much social as it was technical. (LN)." A German student testifies that "Due to the expertise of professionals, a great project manager who kept us on track every week, and the dedication of each team, we were able to do the final product that I am personally proud off".

Regarding their overall experiences, students expressed strong emotions. A male student from Indonesia stated that "this is truly a life-changing experience, although it wasn't smooth sailing all the way." while a German student, exclaimed that " that's something you would normally never think of." Students fully acknowledged the learning opportunity the project provided, be it communication, technical or intercultural. For most of them, it was a chance to improve their professional English skills, as mentioned by a German student: "Within the project, I could improve my English skills and learned how to work with an international team through different time zones."

The students appreciated working in a multicultural team and expanding their personal relations over continents. As expressed by an Indonesian student: "We were getting to know each other and even talking about our countries, such as our culture, food and daily life." The Peruvian female student said "I think the most amazing thing about it is the fact that I've worked with a lot of people from all over the world and we have achieved a very wonderful product for our client."

Besides the disruptions caused by the pandemic the students mentioned a number of challenges with working across time zones as the most prevalent. A Peruvian student stated that: "One of the major challenges of the project was coordinating the team because of time zones. For some of us, that day was just beginning, others were about to have lunch and some others were finishing their day". While another Peruvian student said that "From the beginning, we knew that it would be difficult to reconcile the schedules, since there is a difference of 12 hours with Indonesia, so our weekly coordination meetings and meetings with the students were adapted to that time." This sentiment was further confirmed by one of the German students "I think the most required ability, though, was being agile, adjusting to different time zones, participating sometimes late in the evening, all the morning during the weekends with different time zones and different means of communication." Yet another Peruvian student expressed the challenge of having to deal with multiple issues "I have to manage to overcome the difficulties associated with the pandemic, schedules, language, and, perhaps, specific knowledge of tools, to be able to develop together with students from Germany, Namibia, and Indonesia". One of the female students was concerned with having been the only female in a team and her challenges of being heard and respected. 


\section{Reflections and conclusion}

In conclusion, the GIPE project experience was enriching in many ways: culturally, academically, and professionally. Reviewing the sentiments expressed by the students confirms observations made by the guides, that though the students experienced many new challenges they equally appreciated the learning and the multicultural context. Working in an interdisciplinary team on a software development project required a steep learning curve in a short time. The students received structured technical training and were exposed to new web programming frameworks, and learned good project management practices using professional tools. An invaluable reward was the creation of new personal and professional relations during a time of "social distancing". The Covid-19 pandemic drastically changed the implementation of the planned GIPE framework, depriving the students from travelling to Germany and experience a two-week team-building workshop, as well as depriving the German students from handing over the project to a client on another continent. Yet the distributed interdisciplinary project was completed successfully with a multicultural team of students from four continents, serving as a proof of concept.

We postulate that integrating such international projects in existing curricula across the globe promotes the acquisition of 21 st-century skills for students from all disciplines. Although GIPE received funding for a period of four years only, it is intended to be continued and sustain once the processes have been established and the value for all participating institutions has been recognized. The coordination of such international educational collaborations, however, requires consideration of (1) formal university structures such as lecturing periods, curriculum integration, university calendars, and crediting systems; (2) human resource intensive project preparations, planning and management; (3) communication dynamics and challenges, and the facilitation of peer-instruction and team guidance; (4) technical and methodological aspects of distributed solution development, such as agile development, client interactions, and state of the art development tools and technology implementations. Based on our experiences, we conclude that a refined concept of this interdisciplinary, international project-based learning is a promising approach to support global educational development, even during pandemic times.

\section{Acknowledgements}

The GIPE Project is funded by the German Federal Ministry for Education and Research (BMBF) through the German Academic Exchange Service (DAAD) under grant no. 57510482.

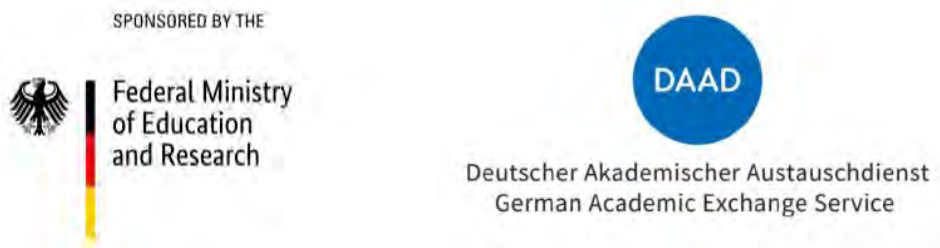

\section{References}

Appiah-Kubi, P. \& Annan, E. (2020). A Review of a Collaborative Online International Learning. International Journal of Engineering Pedagogy (iJEP), 10 (1), 109-124

Bender, W. (2012). Project-Based Learning: Differentiating Instruction for the $21^{\text {st }}$ Century. Thousand Oaks, CA, USA: Corwin Press.

Brereton, P. \& Gumbley, M. \& Lees, S. (1998). Distributed Student Projects in Software Engineering. In Conference on Software Engineering Education and Training (4-22), Los Alamitos, CA, USA: IEEE Computer Society.

De Wit, H. (2013). COIL - Virtual mobility without commercialization. University World News. Retrieved from https://www.universityworldnews.com/post.php?story=20130528175741647

DePaul University (2017). Global Learning Conference: Transcending Boundaries Through Collaborative Online International Learning (COIL). Retrieved from http://www.globallearningconference.org/

Helm, F. \& O'Dowd, R. (2020). Virtual Exchange and its role in Blended Mobility Initiatives. UNICollaboration Position Paper. Retrieved from https://www.unicollaboration.org/wpcontent/uploads/2020/09/Position-paper-on-Blended-Mobility.pdf

SUNY COIL Center (n.d.), Faculty Guide for Collaborative Online International Learning Course Development (vol. 1.4), Center for Collaborative Online International Learning, State University of New York Global Center, Retrieved from http://www.ufic.ufl.edu/uap/forms/coil_guide.pdf 